Modern Physics Letters A

(C) World Scientific Publishing Company

\title{
Towards a Better System for Short Range Precision Force Measurements
}

\author{
Thomas Bsaibes \\ Physics Department, Indiana University - Purdue University Indianapolis, 402 N. Blackford St. \\ Indianapolis, IN 46202, United States of America \\ tbsaibes@iupui.edu \\ Luís Pires \\ Instituto de Física, Universidade Federal do Río de Janeiro, Río de Janeiro, 21941-909, Brazil \\ David Czaplewski \\ Center for Nanoscale Materials, Argonne National Laboratory \\ 9700 S. Cass Avenue, Lemont, IL 60439, United States of America \\ Daniel López \\ Center for Nanoscale Materials, Argonne National Laboratory \\ 9700 S. Cass Avenue, Lemont, IL 60439, United States of America \\ Ricardo S. Decca \\ Physics Department, Indiana University - Purdue University Indianapolis, 402 N. Blackford St. \\ Indianapolis, IN 46202, United States of America \\ rdecca@iupui.edu \\ Received (Day Month Year) \\ Revised (Day Month Year)
}

\begin{abstract}
Many precision experiments have been done in the Casimir regime and in short range gravity when the separation between the interacting bodies is in the sub-micron range. Experimental complexity is minimized when one of the bodies is a sphere and the other one is a plate, making the alignment between the two bodies ubiquitous. Our group has produced the most precise Casimir measurements, and the best limits on predicted Yukawa-like potentials by measuring a force between a $R \sim 150 \mu \mathrm{m}$ sphere attached to a $(500 \mu \mathrm{m})^{2}$ micro-mechanical oscillator and a planar source mass. By replacing the spherical surface with a fraction of a $500 \mu \mathrm{m}$ long cylinder with $R \sim 150 \mu \mathrm{m}$, the force sensitivity can be greatly enhanced. Here, it is paramount to know the angular deviation between the long axis of the cylinder and both the axis of rotation of the oscillator and the plate. Tests between a cylinder and a structure etched into a silicon wafer show that deviations of $20 \mu$ radians are readily accessible. Additionally, a scaled up experiment is used to investigate if capacitance measurements can determine the orientation of the cylinder with respect to a plane with the required precision.
\end{abstract}

Keywords: Short range gravity, Yukawa potential, Casimir, cylinder

PACS Nos.: 04.50.+h, 04.80.Cc, 12.20.Fv, 11.25.Mj

This is the author's manuscript of the article published in final edited form as:

Bsaibes, T., Pires, L., Czaplewski, D., López, D., \& Decca, R. S. (2020). Toward a better system for short range precision force measurements. Modern Physics Letters A, 35(03). https://doi.org/10.1142/S0217732320400027 
2 Thomas Bsaibes et al.

\section{Introduction}

Many theories beyond the standard model predict deviations from Newtonian gravitation at sub-micron separations. ${ }^{1-3,10,11}$ The deviations from Newtonian gravity are characterized by Yukawa-like potentials.

$$
V(r)=-G \frac{m_{1} m_{2}}{r}\left(1+\alpha e^{-r / \lambda}\right)
$$

Where $V(r)$ is the gravitational potential between two point masses $m_{1}$ and $m_{2}$ separated by $r$ and $G$ is is Newton's constant. The first term is the classical Newtonian potential, the second term is the correction with strength $\alpha$ and interaction length $\lambda$. Many experiments have been done to explore these deviations over a large range of $\lambda$ and set constraints in the $\alpha-\lambda$ phase space. ${ }^{4,7-9,12-15,18-20}$

At the time of writing the strongest constraints placed on $\alpha$ in the sub-micron regime was done at Indiana University-Purdue University Indianapolis by the Decca group. ${ }^{4}$ The experiment comprised of a micro-mechanical oscillator with a spherical test mass attached to it, which was brought down to $\sim 200 \mathrm{~nm}$ above a source mass of two alternating material. ${ }^{4}$ However if the test mass is replaced with a fraction of a cylinder the constraints placed on $\alpha$ can be improved by about 2 orders of magnitude over the constraints achieved with a sphere. Figure 1 shows the limits placed by various short range gravity experiments including the limits placed with a spherical test mass (red curve) and the expected improvement from a cylindrical test mass (blue curve).

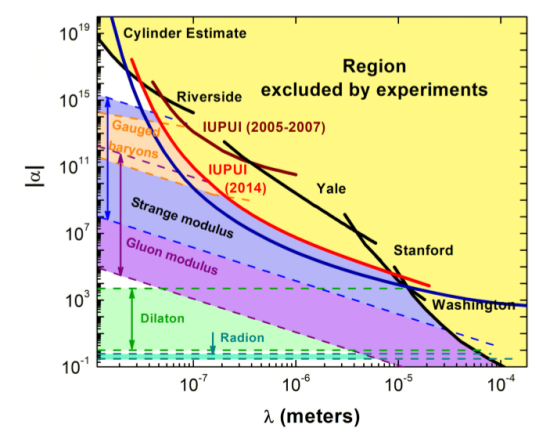

Fig. 1: Experimental limits placed on $\alpha \cdot{ }^{4,14,17-20}$ The red curve is the limits set by IUPUI ${ }^{4}$ and the blue curve is the estimated improvement by using the partial cylindrical test mass.

Similarly, the best results in Casimir physics have been obtained with a similar setup (i.e. a sphere glued to a micro-mechanical oscillator). In this regard there has been an experiment using a cylinder ${ }^{5}$ and theoretical papers indicating how the use of a cylinder would improve on the precision on Casimir physics. ${ }^{6}$ This paper will discuss the techniques used to manufacture the cylindrical test masses and the ability to align the cylinders with an already existing structure. In addition to the cylinder's manufacture process, a capacitance method of determining the cylinder's 
orientation with respect to a plane will be presented.

\section{Cylinder Manufacturing and Alignment}

The cylindrical test masses are being manufactured using a greyscale lithographic technique $^{23}$ with a Heidelberg MLA $150^{21,22}$ at Argonne National Laboratory. Greyscale lithography is a lithographic process which uses a digital pattern to define the desired shape and writes the pattern into photoresist. The cylinder's pattern is made of rectangles $1 \mu \mathrm{m}$ wide and each rectangle has a numerical value associated with it that determines the intensity of the writing laser. By changing the intensity in different locations the cylindrical shape (actually a step-wise approximation to a cylindrical shape) is written into the photoresist. Using this technique will allow for the cylinders to be fabricated directly onto the micro-mechanical oscillators. The goal is to make partial cylinder with a radius of $\sim 150 \mu \mathrm{m}$, a sagitta of $20 \mu \mathrm{m}$ and a length of $500 \mu \mathrm{m}$. The photoresist will be coated with a $10 \mathrm{~nm}$ layer of Ti and a $250 \mathrm{~nm} \mathrm{Au}$ film, in order to be able to perform precision measurements in the Casimir regime and short rage gravity experiments. Figure 2a is a cross section of the cylinder that was made on a silicon wafer; it is a little more than $5 \mu \mathrm{m}$ shorter than expected but the pattern used to make the cylinder can be modified such that the cylinder will have the full $20 \mu \mathrm{m}$ height. The roughness visible in the cylinder can be smoothed out by reflowing the resist before coating them in metal. Reflow is a process where the cylinders are heated causing them to become viscous enough for surface tension to pull the photoresist into a smoother shape, see Fig. 2b. The cylinder in Fig $2 \mathrm{~b}$. was heated at $150{ }^{\circ} \mathrm{C}$ for 1 minute.

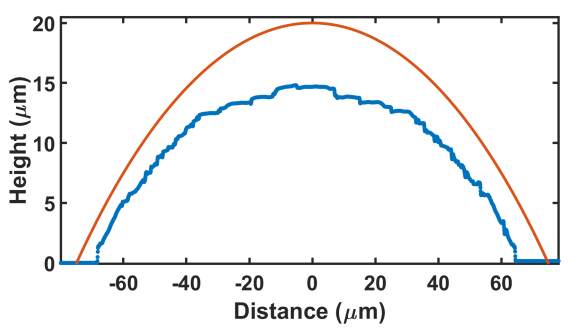

(a)

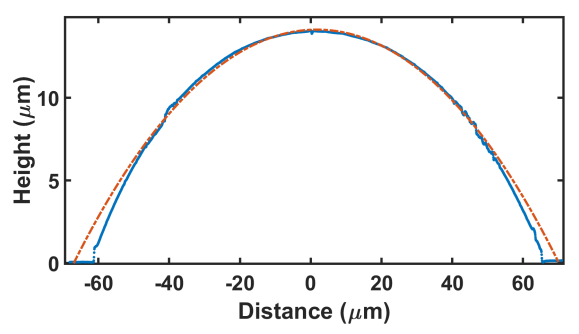

(b)

Fig. 2: a) Cross section of a cylinder. It is shorter than the goal of $20 \mu \mathrm{m}$ (shown in red), which can be adjusted through the pattern that created the cylinder and the roughness can be smoothed with a reflowing process, see text. b) Cross section of a cylinder post reflow using the pattern, used in Fig 2a. The roughness has been reduced substantially and the cross section closely matches the arc of a right circular cylinder (represented by the dashed line). The cylinder was reflowed at 150 ${ }^{\circ} \mathrm{C}$ for 1 minute.

Since the cylinders will be deposited onto an already existing structure, namely the mico-mechanical oscillators, the alignment between the axis of rotation of the oscillator and the long axis of the cylinder needs to be known to a high precision. The 


\section{Thomas Bsaibes et al.}

goal is to achieve an alignment of $1 \mu$ radian, which corresponds to $0.5 \mathrm{~nm}$ deviation over $500 \mu \mathrm{m}$ of the oscillator. In order to determine whether a $1 \mu$ radian separation is achievable, a reference grid was etched into a silicon wafer and a $1 \mathrm{~cm}$ long cylinder was placed next to it, see Fig 3a. The separation between the edge of the grid and the middle of the cylinder is determined using image analysis techniques on images taken with an SEM and a confocal microscope. A canny edge detection algorithm is used through the Fiji distribution of Image $\mathrm{J}^{16}$ to isolate the edges of the etched structure and the cylinder; the separation between the edges was then extracted using scripts built in MATLAB. Figure 3b shows the separation measured between the reference grid and the $1 \mathrm{~cm}$ long cylinder. The deviation is determined by subtracting the separations at two different points along the cylinder and dividing that difference by the distance between those two points. The deviation between the structure and the cylinder is 20 radians over $6 \mathrm{~mm}$ (mainly limited by the optical resolution of the acquired images). From $6 \mathrm{~mm}$ onward the separation decreases, this issue could result from a number of candidates: it is possible that an error occurred in either the etching process, or an error happened while writing the end of the cylinder, or the photoresist at the end of the cylinder is being over developed causing it to be misshapen. Work continues towards identifying the decrease in separation towards the end of the structure.

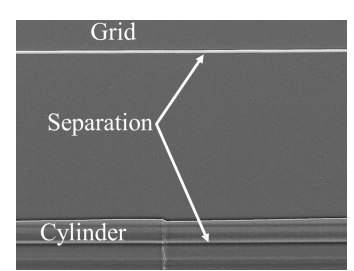

(a)

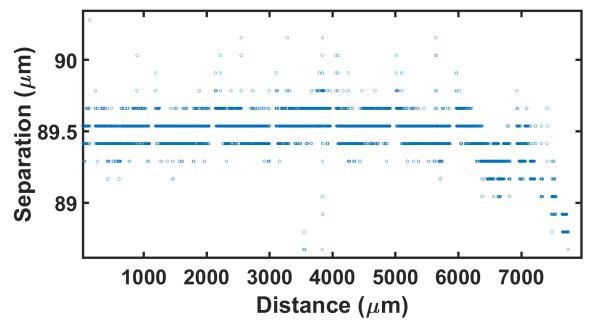

(b)

Fig. 3: (a) A low magnification SEM image of part of the grid and the cylinder, showing what separation is being determined. (b) The separation between the cylinder and the grid. There is a down turn in the separation toward the end of the cylinder. It is not clear whether this is due to a defect in the etched grid or an error in the cylinder processing.

Another approach of determining the separation between two structures is being tested, where high magnification SEM images are taken of just the edge of the grid then moving the stage to take an image of just the cylinder's edge and by knowing the amount that the SEM stage has moved the separation can be worked out. This procedure has been used to achieve a resolution of $40 \mathrm{~nm}$ (yielding a determination of the angle to be better than $2 \mu$ radians), and promises to be improved if an even higher magnification is used. 


\section{Cylinder Orientation}

In order to use the cylinder as test mass in short range gravity experiments, the orientation with respect to the source mass needs to be known. Here a proof of concept test using capacitance to determine the cylinder's orientation and position is described. Once the method is polished, it can be easily extended to the cylinders fabricated in the oscillators (the values of capacitance in the proof of concept are calculated to be similar to the ones observed in the fabricated cylinders). The experimental set up of for the proof of concept consists of a brass cylinder over a brass plate. An optical set up directs a laser down onto either side of the cylinder; the ends of the cylinder are chamfered so mirrors can be placed on the cylinder. The mirrors on the cylinder direct the laser beam to two calibrated position sensitive detectors (PSD) to monitor the cylinder's motion. The cylinder is connected by two rods to a motorized stage that is used to adjust the cylinder with respect to the plate. We are interested in seeing how the capacitance changes as the cylinder's height and tilt along its long axis are changed. Figure 4 shows a schematic of the set up.

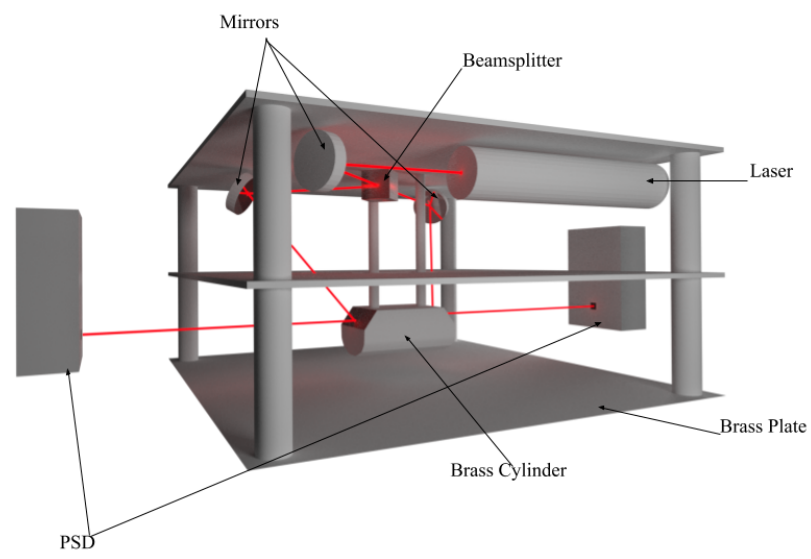

Fig. 4: A schematic of the proof of concept set up to test if capacitance will be a viable alignment indicator. The set up consists of a HeNe laser, 3 mirrors, a beamsplitter, 2 PSD detectors, and a brass cylinder over a brass plate. The ends of the cylinder are cut with a chamfer where mirrors are placed to direct the laser beam to the PSD detectors.

As the cylinder's orientation is changed, the motion of the laser bean along both PSDs are monitored simultaneously. These two sets of measurements are correlated with each other and are used to monitor the cylinders motion. For instance if the laser beam move down in both PDSs the cylinders height is changing and by knowing the change in height on the PSDs the change in height of the cylinder can be calculated with

$$
h=h_{0}+\Delta y_{1}+\Delta y_{2}
$$




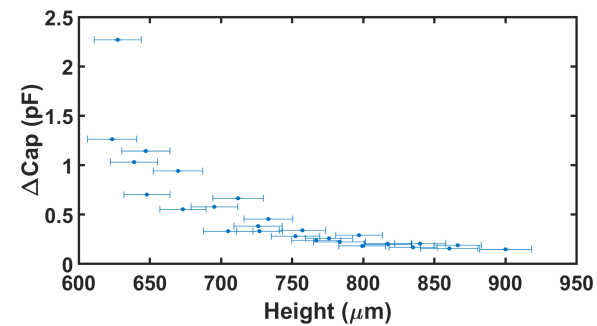

(a)

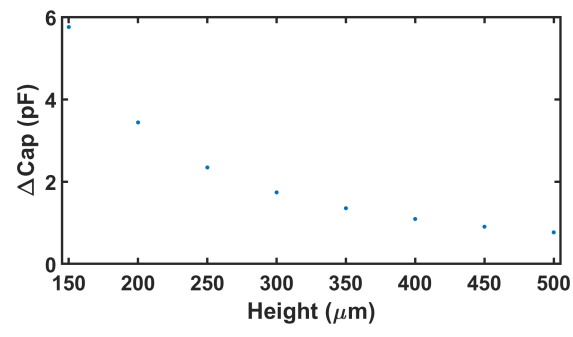

(b)

Fig. 5: a) Preliminary data showing the change in capacitance as a function of the cylinder's height above the plate. The cylinder started at a height of $900 \mu \mathrm{m}$ and it was lowered toward the brass plate. As expected the capacitance increases as the separation between the plate and cylinder decreases. There are vertical error bars present, but they are too small to be visible. b) Simulation data of the change in capacitance as a the height of the cylinder changes.

where $h$ is the height, $h_{0}$ is the initial height determined by stacking shims between the cylinder and the plate and measuring their thickness, and $\Delta y_{1}$ and $\Delta y_{2}$ are the changes along the PSDs. As the height of the cylinder is adjusted the capacitance is recorded. The change in capacitance from one height to the next can then be plotted against the range of $h$ determined from Eq. 2. Figure 5a shows preliminary data of the change in capacitance as a function of height.

For comparison Fig. 5b shows data simulated with Comsol. ${ }^{24}$ The change in capacitance as the height decreases exhibits a similar trend. It should be noted that the range of $h$ in the simulation and in the real data are not the same, but in both the change in capacitance increases as the separation between the plate and the cylinder decreases.

If the PSDs record opposite changes, i.e. $\Delta y_{1}=-\Delta y_{2}$, then the angle of the cylinder is changing and can be calculated from Eq. 3 .

$$
\alpha=\alpha_{0}+\arctan \left(\frac{\Delta y_{1}-\Delta y_{2}}{l_{1}+l_{2}}\right)
$$

where $\alpha$ is the cylinder's tilt, $\alpha_{0}$ is the initial angle, $\Delta y_{1}$ and $\Delta y_{2}$ are again the changes along the PSDs, and $l_{1}$ and $l_{2}$ are the distances from the mirrors to their respective PSD. Figure 6a shows preliminary data of the change in capacitance as a function of angle.

If Fig 6a. is compared to simulation data (Fig. 6b.), it seems that there is again a similar trend. However, the change in capacitance seems much noisier than the change in capacitance seen in Fig. 5a. There are several factors that can contribute the noise, but one known source is the height of the cylinder changes as the angle is adjusted, affecting the capacitance values. More work needs to be done to minimize the sources of noise in the change of angle measurements. 




(a)

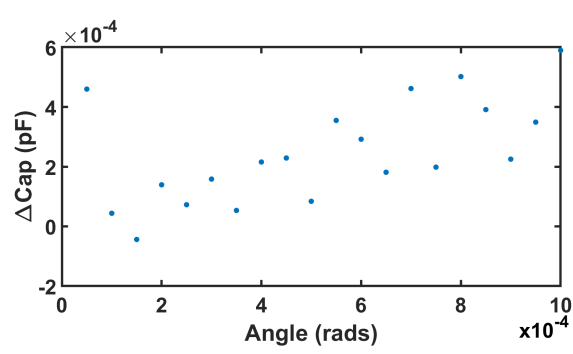

(b)

Fig. 6: a) Preliminary data showing the change in capacitance as a function of angle with respect to the plate. There seems to be a trend comparable to simulation but it is more noisy. b) Simulated change in capacitance at a given angle.

\section{Conclusions}

The cylinders presented in this paper will be used in a short range gravity experiment, from which limits on Yukawa-like potentials will be placed with an expected 2 order of magnitude improvement over the current best limits in the sub-micron regime. They will be also used in Casimir experiments, allowing to extend the range over which Casimir experiments can be precisely performed. We expect that by using cylinders we will be able to obtain meaningful Casimir on separations up to 10 $\mu \mathrm{m}$. Successful measurements depends on the ability to align the cylinder with the axis of rotation of a micro-mechanical oscillator during the cylinder's fabrication process. Current attempts have resulted in an alignment of 20 pradians between a structure etched into a silicon wafer and a deposited cylinder, but work toward bringing the deviation down to 1 rradian continues.

Short range force experiment also hinge on knowing the orientation of the cylinder with respect to a planar source mass. To this end a scaled up proof of concept test is currently underway to show that capacitance can be used as an alignment indicator to know the cylinder's height above the source mass as well as knowing the angle between them. Many improvements are currently being implemented. We expect that the next set of measurements will have the required precision. Under these circumstances the approach will be implemented in the fabricated cylinders on the micro-mechanical oscillators.

\section{Acknowledgments}

Use of the Center for Nanoscale Materials, an Office of Science user facility, was supported by the U.S. Department of Energy, Office of Science, Office of Basic Energy Sciences, under Contract No. DE-AC02-06CH11357. R. S. Decca acknowledges support from the National Science Foundation through grants PHY-1607360 and PHY-1707985 and financial and technical support from the IUPUI Nanoscale Imaging Center, the IUPUI Integrated Nanosystems Development Institute, and the Indiana University Center for Space Symmetries. 


\section{References}

1. B. A. Dobrescu and I. Mocioiu, JHEP 2006, 11 (2006)

2. S. Aldaihan, D. E. Krause, J. C. Long, and W. M. Snow, Phys. Rev. D 95, 096005 (2017)

3. Pavel Fadeev, V. Stadnik, F. Ficek, M. G. Kozlov, V. V. Flambaum, and D. Budker, Phys. Rev. A 99, 022113 (2019).

4. Y. -J. Chen, W. K. Tham, D. E. Krause, D. López, E. Fischbach, and R. S. Decca, Phys. Rev. Lett. 116, 221102 (2016).

5. See, for example, R. Onofrio, N. J. Phys. 8, 237 (2006).

6. R. S. Decca, E. Fischbach, G. L. Klimchitskaya, D. E. Krause, D. López, and V. M. Mostepanenko, Phys. Rev. A 82, 052515 (2010); Phys. Rev. A 84,042502 (2011).

7. J. A. R. Cembranos, A. L. Maroto and H. Villarrubia-Rojo, JHEP 2017, 9 (2017).

8. W. Tan, S. Yang, C. Shao, J. Li, A. Du, B. Zhan, Q. Wang, P. Luo, L. Tu, and J. Luo, Phys. Rev. Lett. 116, 131101 (2016).

9. E. G. Adelberger and T. A. Wagner, Phys. Rev. D 88, 031101 (2013).

10. N. Arkani-Hamed, S. Dimopoulos, and G. Dvali, Phys. Rev. D 59, 086004 (1999)

11. S. Winberg, Phys. Rev. Lett. 40223 (1978)

12. F. M. Piegsa and G. Pignol, Phys. Rev. Lett. 108181801 (2012)

13. J. C. Long and J. C. Price, C R PHYS 4337 (2003)

14. R. S. Decca, D. López, E. Fischbach, G. L. Klimchitskaya, D. E. Krause, and V. M. Mostepanenko, Eur. Phys. J. C 51963 (2007)

15. J. C. Long and V. A. Kostelecký Phys. Rev. D 91092003 (2015)

16. J. Schindelin, I. Arganda-Carreras, E. Frise et al. Nature Methods 9676 (2012)

17. A. Roy and U. Mohideen, Phys. Rev. Lett. 824380 (1999); G. L. Klimchitskaya, A. Roy, U. Mohideen, and V. M. Mostepanenko, Phys. rev. A 603487 (1999); B. W. Harris, F. Chen, and U. Mohideen, Phys. Rev. A 62052109 (2000).

18. A. O. Sushkov, W. J. Kim, D. A. R. Dalvit, and S. K. Lamoreaux, Phys. Rev. Lett. 107, 171101 (2011).

19. A. A. Geraci, S. J. Smullin, D. M. Weld, J. Chiaverini, and A. Kapitulink, Phys. Rev. D 78022002 (2008)

20. D. J. Kapner, T. S. Cook, E. G. Adelberger, J. H. Gundlach, B. R. Heckel, C. D. Hoyle, and H. E. Swanson, Phys. Rev. Lett. 98021101 (2007)

21. The Maskless Aligner MLA150 - now also in a high-resolution version. (0AD). Retrieved from https://www.himt.de/index.php/MLA150.html

22. S. Diez, Proc. SPIE 9761976102 (2016)

23. J. H. Lake, K. M. Walsh, S. McNamara IEEE 978-1-4244-4193-8 1889 (2009)

24. COMSOL Multiphysics $\AA$ v. 5.4. www.comsol.com. COMSOL AB, Stockholm, Sweden. 